\title{
Uma análise sobre o desenvolvimento participativo de jogos educacionais voltados para a terceira idade
}

\author{
Ezequiel Duque $^{1}$, Michelle Nery ${ }^{1}$, Guilherme Fonseca ${ }^{1}$ \\ Felipe Soares $^{1}$, Hebert Pereira ${ }^{1}$, Lucila Ishitani ${ }^{1}$ \\ ${ }^{1}$ Pontifícia Universidade Católica de Minas Gerais (PUC Minas) \\ Rua Walter Ianni, 255 - São Gabriel - Belo Horizonte - MG - Brasil \\ \{ezequiel.duque, falsoares\}@sga.pucminas.br, lucila@pucminas.br \\ \{micnery, guilhermeitabayana, hebert.pereira.dev\}@gmail.com
}

\begin{abstract}
In Brazil, the elderly population (60 years or more) grew by 4.8 million from 2012 to 2017. With the increased life expectancy, it is important to create applications for older people to continue to learn and be able to keep up with the changes. This article aims to verify the contributions that the elderly could provide in the process of developing educational games for their age group if Participatory Design was used. Adopting a qualitative methodology, data collection was based on interviews and data analysis, in the Affinity Diagram. The results show the elderly can contribute and should be involved in the process of developing educational games that meet their needs.
\end{abstract}

Resumo. No Brasil, a população de idosos (60 anos ou mais) cresceu 4,8 milhões de 2012 até 2017. Com o aumento da expectativa de vida, é importante criar aplicações para que idosos continuem a aprender e consigam acompanhar as mudanças. Este artigo tem como objetivo verificar as contribuições que o idoso poderia fornecer no processo de desenvolvimento de jogos educacionais para sua faixa etária, caso o Design Participativo fosse utilizado. Adotando uma metodologia qualitativa, a coleta de dados baseou-se em entrevistas e a análise de dados, no Diagrama de Afinidade. Os resultados mostram que o idoso pode contribuir e deve ser envolvido no processo de desenvolvimento de jogos educacionais que atendam suas necessidades.

\section{Introdução}

De acordo com o estatuto do idoso vigente no Brasil [Brasil 2003], as pessoas com idade igual ou superior a 60 anos são consideradas idosas. Neste trabalho, serão considerados sinônimos de idosos os termos "terceira idade" e "adultos mais velhos". A população idosa brasileira cresceu 4,8 milhões de 2012 até 2017 e supera a marca dos 30,2 milhões de pessoas [IBGE 2017], em função desse aumento, é importante oferecer mais opções de atividades de aprendizagem e lazer para a terceira idade.

Para que idosos possam aprender melhor, o ambiente educacional deve incentivar o questionamento e apresentar desafios. É importante buscar embasamento na gerontagogia, que para proporcionar a aprendizagem entre adultos idosos, estuda o processo de envelhecimento humano com atenção às necessidades físicas, emocionais e sociais que surgem com a idade [Machado et al. 2011]. 
VII Congresso Brasileiro de Informática na Educação (CBIE 2018)

Anais do XXIX Simpósio Brasileiro de Informática na Educação (SBIE 2018)

Ainda segundo a gerontagogia, a participação do idosos é um fator primordial no processo de aprendizagem, sendo importante trabalhar com aspectos motivacionais por eles elegidos como significativos [Machado et al. 2011]. Em aplicações criadas com o foco na terceira idade, metodologias como o design centrado no usuário (DCU) e o design participativo (DP) têm sido utilizadas ao longo do processo de desenvolvimento [Kopeć et al. 2017, Bossen et al. 2013, Iacono and Marti 2014, Santos et al. 2016]. Essas metodologias incentivam a participação do público-alvo no processo de desenvolvimento de uma aplicação.

Os jogos educacionais podem unir entretenimento e aprendizado para os idosos [Ferreira and Ishitani 2015]. O principal objetivo deste trabalho foi identificar as contribuições que o jogo teria, caso o idoso estivesse no processo de desenvolvimento com utilização de metodologias como o DCU e o DP.

Nesta pesquisa, utilizou-se a metodologia qualitativa, com características descritivas. A coleta de dados foi baseada no uso de instrumentos como o questionário e a entrevista. Os procedimentos de análise dos dados se basearam na criação de um Diagrama de Afinidade. Os resultados demonstram que os idosos devem participar do processo de desenvolvimento para reforçar os requisitos considerados a partir da literatura e para encontrar novos requisitos.

Este artigo está estruturado da seguinte forma: na Seção 2 são discutidos trabalhos relacionados a esta pesquisa; na Seção 4 é descrito o método da pesquisa desenvolvido para a elaboração deste trabalho; na Seção 5 é apresentada uma discussão sobre o Diagrama de Afinidade criado com base nos dados coletados; por fim, na Seção 6 são apresentadas as conclusões deste trabalho.

\section{Revisão de literatura}

Nesta seção serão apresentados e discutidos os principais conceitos e trabalhos identificados na revisão de literatura.

\subsection{Jogos educacionais para a terceira idade}

Jogos digitais podem contribuir para retardar o processo de envelhecimento das pessoas, pois podem amenizar as restrições decorrentes da idade [Shang-Ti et al. 2012]. Para tal, devem respeitar critérios de acessibilidade, usabilidade e jogabilidade. Quando o jogo não respeita esses critérios, os idosos não conseguirão utilizá-lo, devido às suas limitações físicas e cognitivas, que podem ser: perda de memória, de atenção, de imaginação, de associação, de percepção e de raciocínio [Foukarakis et al. 2011].

A pesquisa de Nogueira and Ishitani (2012) realizou um levantamento para identificar as características que os jogos digitais para smartphones devem ter para que os idosos sintam o prazer e o desejo de usá-los. O trabalho ressalta que jogos para idosos devem ser projetados com características que motivem os idosos a jogarem, contribuindo assim para o desenvolvimento da aprendizagem combinada com o entretenimento. Dentre os interesses e as expectativas citadas está o aprendizado. Os idosos esperam que as aplicações os mantenham atualizados, fornecendo-lhes meios de aprendizagem, entretenimento, interatividade e de se comunicarem com as outras pessoas [Nogueira and Ishitani 2012].

Porém, mesmo com todos benefícios citados anteriormente e aumento da população idosa, existe uma carência de publicações sobre jogos digitais edu- 
VII Congresso Brasileiro de Informática na Educação (CBIE 2018)

Anais do XXIX Simpósio Brasileiro de Informática na Educação (SBIE 2018)

cacionais, que considerem as limitações cognitivas e expectativas do público idoso [Ferreira and Ishitani 2015].

\subsection{Design centrado no usuário idoso}

O termo Design Centrado no Usuário (DCU), do inglês User Centered Design, foi originado por Don Norman em 1980 [Norman and Draper 1986]. DCU é definido como uma estrutura de um processo de design que aumenta a usabilidade e a aceitação de um sistema [ISO 2010]. Assim como na DCU, o Design Participativo (DP), do inglês Partcipatory Design, se concentra nas opiniões dos usuários para criar projetos apropriados [Lee et al. 2017]. A metodologia do DP enfatiza o envolvimento do usuário, idealmente durante todo o ciclo de vida do projeto, desde a geração inicial do conceito até o processo de desenvolvimento de tecnologia e avaliação [Bossen et al. 2013].

O uso de métodos, como DCU e DP, é uma maneira de desenvolver as soluções certas para um público-alvo específico [Hornung et al. 2017]. O público idoso tem dificuldade para interagir e utilizar determinadas tecnologias e a falta de envolvimento dos idosos nas etapas de concepção e avaliação do desenvolvimento de produtos pode ser um dos motivos [Wilkinson and Angeli 2014]. O uso de DCU e DP para o desenvolvimento de aplicações para idosos tem sido um fator positivo [Ferreira et al. 2014, Kopeć et al. 2017, Wilkinson and Angeli 2014]. Ao envolver esse público-alvo, os desenvolvedores aumentam a possibilidade de criar um produto que considera limitações e características específicas dos adultos mais velhos [Ferreira et al. 2014].

\section{Labuta Batuta}

O jogo Labuta Batuta é do gênero educacional, casual e retrata o cotidiano de um idoso em sua casa, em busca de lazer e comunicação, por meio de uma das tecnologias mais usadas atualmente: um aparelho smartphone.

Primeiramente, o jogador constrói o seu avatar. Em seguida, o jogador pode navegar pelos ambientes virtuais da casa, usando o smartphone para tirar fotos, enviar mensagens, fazer ligações, instalar e jogar minijogos. Existem seis minijogos, que além de divertir, servem para explicar alguns gestos usuais no uso de smartphones, como: executar um zoom em fotos, tocar a tela, arrastar ícones entre outros. Por fim, conteúdos sobre tecnologias atuais são apresentados também por meio dos minijogos, como palavras específicas usadas na internet, emoticons, redes sociais, gírias comuns e abreviações usadas em mensagens de texto.

No trabalho de Ferreira et al. (2015) o Labuta Batuta foi utilizado para identificar características necessárias aos jogos educacionais em favor do aprendizado de adultos mais velhos, os autores concluíram que os adultos mais velhos aprendem ao jogar um jogo móvel específico para eles. A análise quantitativa evidenciou que após jogarem o jogo, o número de acertos por questão aumentou em uma avaliação de conhecimentos realizada [Ferreira et al. 2015]. Além disso, os idosos melhoraram a proficiência na execução de tarefas no celular (tirar fotos, atender chamadas, baixar aplicativos) [Silva et al. 2015].

O processo de criação do jogo não considerou o envolvimento do idoso durante o processo de desenvolvimento com utilização de DCU e DP. O projeto foi dividido em duas fases: a primeira constituiu no levantamento do conteúdo a ser transmitido pelos minijogos e a segunda nos métodos de avaliação e instrumentos que permitiriam elucidar 
VII Congresso Brasileiro de Informática na Educação (CBIE 2018)

Anais do XXIX Simpósio Brasileiro de Informática na Educação (SBIE 2018)

as dificuldades, satisfações e observações dos participantes sobre a experiência de utilizar o aplicativo e os minijogos [Silva et al. 2015].

\section{Metodologia}

Esta seção irá apresentar a metodologia adotada na realização deste trabalho. Para verificar as contribuições que o usuário idoso pode fornecer no processo de desenvolvimento de jogos educacionais, o Labuta Batuta foi escolhido como objeto de teste, pois o seu processo de desenvolvimento não teve participação do idoso. Além disso foram realizadas as seguintes atividades: i) seleção de jogos, para que os participantes interajam e conheçam estilos diferentes de jogos; ii) seleção dos participantes idosos que atendiam aos critérios da pesquisa; iii) coleta de dados por meio de entrevistas e questionários; iv) análise dos dados com base no Diagrama de Afinidade.

\subsection{Seleção dos Jogos}

Além de utilizar o Labuta Batuta foram utilizados mais 3 jogos. Esses jogos foram importantes porque nem todos os participantes jogam ou conhecem mais de um estilo de jogo. Com isso, eles puderam avaliar e verificar se surgiriam outras características que consideram positivas ou negativas. Foi decidido que seriam utilizados jogos casuais, pois de acordo com [Santos and Ishitani 2014] estes são mais adequados ao público idoso, por oferecerem uma opção de entretenimento, diversão e exercícios mentais. Os jogos escolhidos foram: i) Can You Escape; ii) Jogo do Bilhão; iii) Minion Rush, disponíveis na PlayStore ${ }^{1}$.

\subsection{Seleção dos Participantes}

Foram selecionados sete participantes, sendo quatro mulheres e três homens. A estratégia utilizada para conseguir os participantes foi a "bola de neve" [Biernacki and Waldorf 1981]. Os participantes encontrados atenderam aos seguintes critérios: possuir no mínimo 60 anos e ser alfabetizado. Não era necessário ter experiência de jogar em smartphone e possuir um aparelho celular, pois este poderia ser cedido temporariamente pelo grupo de pesquisa.

\subsection{Coleta de dados}

A coleta de dados foi dividida em duas etapas. Inicialmente o participante respondeu a um questionário demográfico que coletou informações referentes à idade, sexo, nível de escolaridade e experiência prévia com jogos. Todos os instrumentos utilizados foram aprovados pelo Comitê de Ética em Pesquisa.

A primeira etapa teve duração de cinco dias. Nela, o participante utilizou um smartphone para jogar Can You Escape, Jogo Bilhão e Minion Rush. O número de sessões, o horário e a duração de cada sessão ficou a cargo do participante. Ao final dos cinco dias foi realizada uma entrevista que buscou: i) a opinião dos idosos em relação aos jogos utilizados; ii) aspectos positivos e negativos dos jogos.

A segunda etapa durou cinco dias e o participante utilizou um smartphone para jogar o Labuta Batuta. Durante esse período o participante pôde fazer anotações sobre

\footnotetext{
${ }^{1}$ Disponível em: https://play.google.com/store. Acesso em: maio de 2018.
} 
VII Congresso Brasileiro de Informática na Educação (CBIE 2018)

Anais do XXIX Simpósio Brasileiro de Informática na Educação (SBIE 2018)

as impressões do jogo. Como é comum que as pessoas mais velhas tenham déficit de memória, as anotações foram sugeridas para ajudá-lo a recordar suas percepções durante a entrevista. Assim como na primeira etapa, o número de sessões de jogo, o horário e a duração de cada sessão ficou a cargo do participante e uma entrevista final foi realizada para coletar: i) característica dos outros jogos testados ou de outros jogos já conhecidos que o participante gostaria de encontrar no Labuta Batuta; ii) o que o participante não gostou no Labuta Batuta; iii) o que, na visão do participante, o Labuta Batuta deveria ter para ensiná-lo a utilizar melhor o celular.

\subsection{Análise dos dados}

Para analisar os dados coletados foi criado um Diagrama de Afinidade. O Diagrama de Afinidade é uma técnica utilizada para organizar e compreender dados qualitativos [Jokela and Lucero 2014]. O objetivo é organizar os dados por meio do agrupamento de ideias, para possibilitar a identificação de padrões e uma melhor compreensão de um problema [Høiseth et al. 2013]. A criação foi realizada no Trello ${ }^{2}$ e de forma colaborativa com a participação de seis pesquisadores. Na Tabela 1 são apresentadas as etapas realizadas para o desenvolvimento do Diagrama.

Tabela 1. Etapas de criação do Diagrama de Afinidade

\begin{tabular}{|l|l|}
\hline Etapas realizadas & Descrição \\
\hline Etapa 1: & $\begin{array}{l}\text { Cada pesquisador analisou os dados coletados pelas entrevistas e } \\
\text { questionários. Foram criados vários "cartões" no Trello, que repre- } \\
\text { sentaram ideias e observações feitas pelos pesquisadores. }\end{array}$ \\
\hline $\begin{array}{l}\text { Etapa 2: } \\
\text { Agrupamento de ideias }\end{array}$ & $\begin{array}{l}\text { De forma aleatória, um "cartão" foi selecionado para fazer parte de } \\
\text { um primeiro grupo, que no Trello é chamado de Quadro. } \\
\text { Para o "cartão" seguinte, foi feita a seguinte análise: "Isso é se- } \\
\text { melhante ao primeiro ou diferente?" Se semelhante, colocá-lo no } \\
\text { mesmo Quadro, caso contrário era criado um novo Quadro. } \\
\text { Esse processo foi realizado até que todos os "cartões" fossem anali- } \\
\text { sados e agrupados dentro dos Quadros. }\end{array}$ \\
\hline $\begin{array}{l}\text { Etapa 3: } \\
\text { Eliminação de dados repetidos }\end{array}$ & $\begin{array}{l}\text { Para que o diagrama ficasse mais objetivo foram eliminados } \\
\text { "cartões" repetidos ou que transmitissem ideias semelhantes. }\end{array}$ \\
\hline Etapa 4: & $\begin{array}{l}\text { Os pesquisadores debateram a respeito de cada Quadro e seus res- } \\
\text { pectivos “cartões", com intuito de aperfeiçoar o diagrama. }\end{array}$ \\
\hline
\end{tabular}

Fonte: Elaborado pelo autor

\section{Resultados}

Nesta seção serão apresentados os resultados obtidos com a criação do Diagrama de Afinidade resultante.

\subsection{Resultados da Construção do Diagrama}

A construção da Diagrama se baseou na realização de quatro etapas. Na primeira etapa foram consideradas todas as entrevistas realizadas, isso quer dizer que nem todos os cartões estão diretamente relacionados ao Labuta Batuta, mas indicam pontos positivos e negativos que podem ser considerados em qualquer jogo para idosos. Na Tabela 2 são apresentados os resultados obtidos após a realização de cada uma das etapas da construção do diagrama.

\footnotetext{
${ }^{2} \mathrm{https}: / /$ trello.com/
} 
VII Congresso Brasileiro de Informática na Educação (CBIE 2018)

Anais do XXIX Simpósio Brasileiro de Informática na Educação (SBIE 2018)

Tabela 2. Resultados de cada etapa da criação do Diagrama

\begin{tabular}{|l|l|}
\hline Etapas & Descrição \\
\hline Etapa 1 & Transcrição dos 83 “cartões” para o Trello. \\
\hline Etapa 2 & Agrupamento dos 83 “cartões” em cinco Quadros. \\
\hline Etapa 3 & Eliminação de 53 “cartões” repetidos ou semelhantes. \\
\hline Etapa 4 & Refinamento gerando diagrama com seis Quadros e 30 “cartões”. \\
\hline
\end{tabular}

Fonte: Elaborado pelo autor

Os seis Quadros ou categorias identificadas foram: Familiaridade, Falta de experiência com jogos, Características que não gostou, Falta de experiência com a Tecnologia, Características que gostou e Falta de adequação a idosos. Na Figura 1 é apresentado o Diagrama de Afinidade resultante.

\begin{tabular}{|c|c|c|}
\hline Familiaridade & Falta de experiência com jogos & Características que não gostou \\
\hline Já conhecia jogos parecidos & \multirow{2}{*}{$\begin{array}{l}\text { Precisou de ajuda de terceiros para } \\
\text { entender os jogos }\end{array}$} & \multirow[t]{2}{*}{$\begin{array}{l}\text { Sentiu falta de mais opções de } \\
\text { interação com avatar }\end{array}$} \\
\hline Relembrou bons momentos já vividos & & \\
\hline $\begin{array}{l}\text { Gostou por ter afinidade com os } \\
\text { temas }\end{array}$ & Dificuldade em entender a interface & $\begin{array}{l}\text { Sentiu falta de eventos que melhoram } \\
\text { alguma habilidade temporariamente }\end{array}$ \\
\hline $\begin{array}{l}\text { Falta de experiência com a } \\
\text { Tecnologia }\end{array}$ & \multirow{2}{*}{$\begin{array}{l}\text { Não gostou da forma de caminhar do } \\
\text { personagem (falta de costume em } \\
\text { jogo no celular) }\end{array}$} & $\begin{array}{l}\text { Jogos muito repetitivos: poderia ter } \\
\text { mais níveis, enjoativos e não tem } \\
\text { ação }\end{array}$ \\
\hline Tem dificuldade em usar o celular & & Jogo fácil demais \\
\hline $\begin{array}{l}\text { Não gostou por não ter conhecimento } \\
\text { prévio em tecnologia }\end{array}$ & Não conseguiu passar de fase & Não chama a atenção \\
\hline $\begin{array}{l}\text { Sentimento de confusão com } \\
\text { anúncios e propaaandas }\end{array}$ & \multirow{2}{*}{$\begin{array}{l}\text { Falta de adequação a idosos } \\
\text { Difícil acertar o movimento } \\
\text { correto(tempo de resposta) }\end{array}$} & \multirow{3}{*}{$\begin{array}{l}\text { Não gostou do Avatar velho demais } \\
\text { O Jogo não motivou o jogador a } \\
\text { permanecer jogando }\end{array}$} \\
\hline Características que gostou & & \\
\hline $\begin{array}{l}\text { Gostou da história e do enredo do } \\
\text { jogo }\end{array}$ & Poderia ter mais pistas & \\
\hline Achou muitas novidades nos jogos & \multirow{2}{*}{$\begin{array}{l}\text { Dificuldade para entender jogos } \\
\text { rápidos e complexos, com muitas } \\
\text { informações }\end{array}$} & \multirow{2}{*}{$\begin{array}{l}\text { Dificuldade com jogos em outros } \\
\text { idiomas (inglês, espanhol) } \\
\text { Deveria informar o "estágio" do jogo. } \\
\text { Ex: em que fase se encontra }\end{array}$} \\
\hline $\begin{array}{l}\text { Sentia-se desafiada com as perguntas } \\
\text { de forma positiva }\end{array}$ & & \\
\hline $\begin{array}{l}\text { Sentimento de emoção ao acertar as } \\
\text { respostas }\end{array}$ & $\begin{array}{l}\text { Difícil acompanhar a velocidade do } \\
\text { jogo }\end{array}$ & Ex: em que fase se encontra \\
\hline $\begin{array}{l}\text { Jogos devem trabalhar com raciocínio } \\
\text { e concentração }\end{array}$ & $\begin{array}{l}\text { Acha que o jogo poderia ter mais } \\
\text { ajuda.(help) }\end{array}$ & conseguir superar o desafio dos jogos \\
\hline
\end{tabular}

Figura 1. Diagrama de Afinidade

\subsection{Discussão}

As informações representadas no Diagrama de Afinidade evidenciam características positivas e negativas na visão do idoso em relação aos jogos utilizados nesta pesquisa. Algumas dessas características já são conhecidas e publicadas, e foram consideradas no projeto de construção do Labuta Batuta. Outras características não foram encontradas na literatura e, por isso, não foram consideradas no projeto do Labuta Batuta, mas devem ser consideradas na construção de qualquer jogo para o público idoso, além de orientarem futuras melhorias no Labuta Batuta. 
VII Congresso Brasileiro de Informática na Educação (CBIE 2018)

Anais do XXIX Simpósio Brasileiro de Informática na Educação (SBIE 2018)

Foram identificados aspectos positivos pelos idosos nos Quadros Familiaridade e Características que gostou. Os participantes relataram: satisfação ao identificar jogos parecidos com aqueles que já conheciam, relembraram bons momentos com a família, se sentiram confortáveis ao lidar com temas que já tinham afinidade e citaram características (desafios, emoção, concentração) que reforçam requisitos apontados na literatura. Portanto, os Quadros Familiaridade e Características que gostou apontam características que de modo geral são importantes para desenvolvimento de jogos com foco na terceira idade. Esses aspectos haviam sido considerados no desenvolvimento do Labuta Batuta.

O Quadro Falta de experiência com a Tecnologia não apresenta novidades em relação a literatura, com exceção do cartão Sentimento de confusão com anúncios e propagandas, pois o Can You Escape e o Jogo do Bilhão possuem muitos anúncios pop-up e involuntariamente o jogador saía do jogo e tinha dificuldades em retornar. O Labuta Batuta não tem nenhum tipo de anúncio que atrapalhe a jogabilidade. Portanto, jogos para idosos devem evitar anúncios durante uma sessão, uma vez que o jogador terá dificuldades de interagir com anúncio pela falta de experiência com a tecnologia.

No Quadro Falta de experiência com jogos está o cartão Não gostou da forma de caminhar do personagem (falta de costume em jogo no celular) que indica que um participante da pesquisa não gostou da forma de interação com o personagem do jogo. Essa referência é feita ao personagem do Labuta Batuta e pode ser uma característica a ser revisada no game. O fato de existir dificuldade na interação com o personagem pode desmotivar o jogador e um novo tipo de interação poderia ser implementada. Os cartões Dificuldade em entender a interface e Precisou de ajuda de terceiros para entender os jogos também possuem relação com o Labuta Batuta e apesar da interface ter sido construída com base em requisitos da literatura, mostra que o idoso ainda pode ter dificuldades de entendimento, cuja causa vai além da falta de experiência com a tecnologia e com jogos. Seguindo essa linha de raciocínio, o cartão Poderia ter mais pistas do Quadro Falta de adequação a idosos e o cartão Acha que o jogo poderia ter mais ajuda (help) reforçam essa necessidade dos idosos. A falta de informação sobre as ações que devem ser feitas pelo jogador pode comprometer a maneira e o tempo do jogador no game. De acordo com Koster (2005) um bom jogo é aquele que ensina tudo o que tem para oferecer, antes que o jogador pare de jogar. Ainda de acordo com o autor, os jogos “(...) são exercícios para nossos cérebros. Jogos que falham em exercitar nossos cérebros se tornam tediosos" [Koster 2005](2005, p.38). A falta de pistas para as ações que devem ser executadas compromete, na opinião dos participantes, o processo de aprendizagem e torna o jogo cansativo. No jogo Labuta Batuta há referência a essas dificuldades encontradas por jogadores idosos conforme registros feitos nos diários dos participantes “(...) Deveria ter tido mais textos, para eu entender mais o jogo.”. Essas dificuldades podem ser minimizadas com a participação do idoso no processo de desenvolvimento e possibilitam um refinamento na interface do jogo. Em um outro relato, um dos participantes ressaltou de maneira positiva o fato de conhecer as ações a serem executadas para solucionar os desafios ou tarefas impostas pelo jogo “(...) Me senti que sou capaz de jogar. Não dá vontade de parar" [Silva et al. 2015].

No Diagrama de Afinidade também existem apontamentos de características que não foram consideradas no desenvolvimento do Labuta Batuta. No Quadro Característica que não gostou o cartão Não gostou do Avatar velho demais mostra que o idoso 
VII Congresso Brasileiro de Informática na Educação (CBIE 2018)

Anais do XXIX Simpósio Brasileiro de Informática na Educação (SBIE 2018)

pode não se sentir representado por um Avatar com características de uma pessoa idosa. Como o Avatar do Labuta Batuta é uma pessoa velha, uma possível melhoria no jogo seria remodelar o personagem de acordo com a opinião e participação direta do idoso na criação do personagem. O Cartão Dificuldade com jogos em outros idiomas (inglês, espanhol), indica dificuldades do participante em jogar um game em que as instruções e contexto estão em inglês. Esta característica não está na literatura como recomendação para jogos com foco na terceira idade e deve ser considerada em projetos futuros. No caso deste trabalho, o inglês estava presente no Can You Escape. O Labuta Batuta não tem esse problema, uma vez que a linguagem utilizada é o português.

Por fim, no cartão Deveria informar o "estágio" do jogo. Ex: em que fase se encontra percebe-se claramente a necessidade de um feedback direto e imediato às ações do jogador. Csikszentmihaly afirma que tudo o que o jogador fizer deve ter uma resposta e isso deve ocorrer para qualquer ação do jogador [Csikszentmihaly 1990]. Ou seja, o jogo deve indicar de forma clara se o jogador executou uma ação de forma correta ou não, permitindo que ele ajuste sua estratégia enquanto joga. Isso provoca no jogador uma maior atenção, favorecendo o surgimento de uma maior concentração e foco, consequentemente uma maior imersão.

\section{Considerações finais}

Esta pesquisa teve como objetivo verificar as contribuições que um idoso poderia oferecer no processo de desenvolvimento de um jogo móvel educacional com utilização de metodologias como o DCU e o DP. Quatro jogos foram utilizados como objeto de teste e, para entendimento do problema, foi construído um Diagrama de Afinidade. Esse Diagrama apresenta resultados genéricos para desenvolvimento de jogos educacionais voltados para os idosos. Dentre essas características, várias ainda não estavam presentes na literatura.

Os resultados reforçam a importância de envolver o idoso no processo de desenvolvimento de um jogo educacional, com utilização do DCU ou DP, para melhor compreensão e adequação dos requisitos levantados e para identificar novos requisitos e funcionalidades para o jogo. Eles também indicam que os idosos podem contribuir no aprimoramento de características já existentes no jogo.

Ao participar do processo de desenvolvimento, o idoso pode contribuir para que o jogo seja divertido e consequentemente proporcione aprendizado ao jogar. De acordo com a literatura, diversão é um dos principais fatores que o jogador busca em um jogo e pode ser relacionada ao aprendizado do jogador com os desafios impostos pelo jogo. Nesse contexto, jogos envolvem um processo de aprendizagem pois estimulam o jogador a desenvolver sua capacidade de concentração e raciocínio em função dos desafios progressivos que lhe são apresentados. Atualmente, jogos são utilizados em treinamentos corporativos, gestão pública, na área da saúde, em escolas de línguas estrangeiras. Por isso, diante da necessidade de atualização constante para acompanhar as inovações, bem como a dificuldade de saber como oferecer mais oportunidade de aprendizado ao público idoso, os jogos são um importante objeto de aprendizagem para esse público.

Como sugestão para trabalhos futuros, sugere-se a adequação do Labuta Batuta com as considerações feitas pelos idosos e que o processo de desenvolvimento de jogos educacionais para idosos utilizem as metodologias DCU e DP. 
VII Congresso Brasileiro de Informática na Educação (CBIE 2018)

Anais do XXIX Simpósio Brasileiro de Informática na Educação (SBIE 2018)

\section{Agradecimentos}

O presente trabalho foi realizado com apoio da Coordenação de Aperfeiçoamento de Pessoal de Nível Superior - Brasil (CAPES) - Código de Financiamento 001.

\section{Referências}

Biernacki, P. and Waldorf, D. (1981). Snowball sampling: Problems and techniques of chain referral sampling. Sociological methods \& research, 10(2):141-163.

Bossen, C., Christensen, L. R., Grönvall, E., and Vestergaard, L. S. (2013). Carecoor: Augmenting the coordination of cooperative home care work. International Journal of Medical Informatics, 82(5):189 - 199.

Brasil (2003). Lei no 10.741, de $1^{\circ}$ de outubro de 2003. http: / / www . planalto. gov.br/ccivil_03/leis/2003/L10.741.htm. Último acesso em 25 de Maio de 2018.

Csikszentmihaly, M. (1990). Flow: The Psychology of Optimal Experience. Harper and Row.

Ferreira, F., Almeida, N., Rosa, A. F., Oliveira, A., Casimiro, J., Silva, S., and Teixeira, A. (2014). Elderly centered design for interaction - the case of the s4s medication assistant. Procedia Computer Science, 27:398 - 408.

Ferreira, R. and Ishitani, L. (2015). Jogos educacionais digitais para idosos: uma revisão sistemática de literatura. In Anais do XXVI Simpósio Brasileiro de Informática na Educação - SBIE 2015, volume 26, pages 404-413.

Ferreira, R., Silva, R. B., Yoshioka, S., Duque, E., Oliveira, G., Nery, M., Mol, A., , Silva, R. S., Álvaro Rocha, and Ishitani, L. (2015). Aprendizagem do uso de smartphones por adultos mais velhos mediada por jogo educacional. In Proceedings of SBGames 2015, pages 945-954, Teresina, PI, Brasil. SBC.

Foukarakis, M., Leonidis, A., Adami, I., Antona, M., and Stephanidis, C. (2011). An adaptable card game for older users. In Proceedings of the 4th International Conference on PErvasive Technologies Related to Assistive Environments, PETRA '11, pages 27:1-27:7, New York, NY, USA. ACM.

Høiseth, M., Giannakos, M. N., and Jaccheri, L. (2013). Research-derived guidelines for designing toddlers' healthcare games. In CHI '13 Extended Abstracts on Human Factors in Computing Systems, pages 451-456, New York, NY, USA. ACM.

Hornung, D., Müller, C., Shklovski, I., Jakobi, T., and Wulf, V. (2017). Navigating relationships and boundaries: Concerns around ict-uptake for elderly people. In Proceedings of the 2017 CHI Conference on Human Factors in Computing Systems, pages 7057-7069, New York, NY, USA. ACM.

Iacono, I. and Marti, P. (2014). Engaging older people with participatory design. In Proceedings of the 8th Nordic Conference on Human-Computer Interaction: Fun, Fast, Foundational, pages 859-864, New York, NY, USA. ACM.

IBGE (2017). Número de idosos cresce 18\% em 5 anos e ultrapassa 30 milhões em 2017. https: / / bit. 1y/2KF JTnM. Último acesso em 25 de maio de 2018. 
VII Congresso Brasileiro de Informática na Educação (CBIE 2018)

Anais do XXIX Simpósio Brasileiro de Informática na Educação (SBIE 2018)

ISO, E. (2010). 9241-210: Human-centered design for interactive systems. Deutsches Institut für Normung.

Jokela, T. and Lucero, A. (2014). Mixednotes: A digital tool to prepare physical notes for affinity diagramming. In Proceedings of the 18th International Academic MindTrek Conference: Media Business, Management, Content \& Services, AcademicMindTrek '14, pages 3-6, New York, NY, USA. ACM.

Kopeć, W., Skorupska, K., Jaskulska, A., Abramczuk, K., Nielek, R., and Wierzbicki, A. (2017). Livinglab pjait: Towards better urban participation of seniors. In Proceedings of the International Conference on Web Intelligence, pages 1085-1092, New York, NY, USA. ACM.

Koster, R. (2005). Theory of Fun for Game Design. Paraglyph Press.

Lee, H. R., Šabanović, S., Chang, W.-L., Nagata, S., Piatt, J., Bennett, C., and Hakken, D. (2017). Steps toward participatory design of social robots: Mutual learning with older adults with depression. In Proceedings of the 2017 ACM/IEEE International Conference on Human-Robot Interaction, pages 244-253, New York, NY, USA. ACM.

Machado, L. R., Maissiat, J., Behar, P. A., and Biazus, M. C. V. (2011). Pedagogia , Andragogia e Gerontologia : utilizando objetos de aprendizagem ao longo da vida. Jornada de Atualização em Informática na Educação, 1:V. 1 N. 1-89-98.

Nogueira, R. and Ishitani, L. (2012). Motivational factors for mobile serious games for elderly users. In Proceedings of SBGames 2012, pages 19-28, Brasilia, DF, Brasil. SBC.

Norman, D. A. and Draper, S. W. (1986). User centered system design; new perspectives on human-computer interaction. L. Erlbaum Associates Inc.

Santos, L. G. N. O. and Ishitani, L. (2014). Uma proposta de heurísticas para avaliação de usabilidade de jogos casuais para dispositivos móveis voltados para idosos. Master's thesis, PUC Minas, Programa de Pós-Graduação em Informática, Belo Horizonte.

Santos, M. M. T., Antonelli, H. L., Rodrigues, S. S., de O. Silva, C. L., Fortes, R. P. M., and Castro, P. C. (2016). Personalizing health-related ict interface and application: Older adults and elderly caregivers preferences. In Proceedings of the 7th International Conference on Software Development and Technologies for Enhancing Accessibility and Fighting Info-exclusion, pages 331-338, New York, NY, USA. ACM.

Shang-Ti, C., Chiang, I.-T., Liu, E. Z.-F., and Chang, M. (2012). Effects of improvement on selective attention: Developing appropriate somatosensory video game interventions for institutional-dwelling elderly with disabilities. TOJET: The Turkish Online Journal of Educational Technology, 11(4).

Silva, R. S., Álvaro Rocha, Ferreira, R., Silva, R. B., Yoshiok, S., Nery, M., Mol, A., and Ishitani, L. (2015). Labuta batuta: um jogo educacional movel para adultos mais velhos. In Proceedings of SBGames 2015, pages 463-472, Teresina, PI, Brasil. SBC.

Wilkinson, C. R. and Angeli, A. D. (2014). Applying user centred and participatory design approaches to commercial product development. Design Studies, 35(6):614631. 